\title{
Higher-Order Co-occurrences for Exploratory Point Pattern Analysis and Decision Tree Clustering on Spatial Data
}

\author{
D.G. Leibovici*,a, L. Bastin ${ }^{\mathrm{b}}$, M. Jackson ${ }^{\mathrm{a}}$ \\ ${ }^{a}$ Centre for Geospatial Sciences, University of Nottingham, UK \\ ${ }^{b}$ School of Engineering and Applied Science, Aston University, UK
}

\begin{abstract}
Analyzing geographical patterns by collocating events, objects or their attributes has a long history in surveillance and monitoring, and is particularly applied in environmental contexts, such as ecology or epidemiology. The identification of patterns or structures at some scales can be addressed using spatial statistics, particularly marked point processes methodologies. Classification and regression trees are also related to this goal of finding "patterns" by deducing the hierarchy of influence of variables on a dependent outcome. Such variable selection methods have been applied to spatial data, but, often without explicitly acknowledging the spatial dependence. Many methods routinely used in exploratory point pattern analysis are second-order statistics, used in a univariate context, though there is also a wide literature on modelling methods for multivariate point pattern processes. This paper proposes an exploratory approach for multivariate spatial data using higher-order statistics built from co-occurrences of events or marks given by the point processes. A spatial entropy measure, derived from these multinomial distributions of co-occurrences at a given order, constitutes the basis of the proposed exploratory methods.
\end{abstract}

Key words: spatio-temporal data, spatial statistics, co-occurrences, multivariate data, marked point process, regression trees, spatial entropy, $\mathrm{R}$

\footnotetext{
* Corresponding author

Email addresses: didier.leibovici@nottingham.ac.uk (D.G. Leibovici),

1.bastin@aston.ac.uk (L. Bastin), Mike.Jackson@nottingham.ac.uk (M. Jackson)
} 
programming

\section{Introduction}

Geographical pattern in experimental datasets is frequently investigated by collocating events, objects or their attributes, particularly when the aim is to monitor the environment, as in ecology or epidemiology (Wagner and Fortin, 2005; Bastin et al., 2007b). In spatial statistics, this is often associated with marked point processes (Diggle, 2003; Schabenberger and Gotway, 2004). In order to identify patterns, or at least to establish the existence of certain structures at some scales, spatial dependence functions, e.g. cross- $K$ functions, may be plotted against distance. These functions can be tested for significance against the functions produced by complete randomness or other hypothetical processes, or against the results of random labelling. Typically, a set of Monte Carlo realisations is used to generate a "null hypothesis" confidence envelope, encompassing, for example, $95 \%$ of the results from the random or hypothesised process (Diggle et al., 2007; Schlather and Diggle, 2004; Lotwick and Silverman, 1982). For a review on recent developments in modelling multivariate spatial processes, see also Baddeley et al. (2006). Classification/Decision/Regression trees such as CART (Breiman et al., 1984), ID3 (Quinlan, 1986), and PEGASE (Phipps, 1981), are also related to the goal of finding "patterns" by selecting and prioritising variables according to their hierarchy of influence on a dependent outcome or on a specific statistic. Often these methods have been applied, without any adaptation, to spatial data, though some authors propose specific modifications to account for spatial dependence (Bel et al., 2009; Li and Claramunt, 2006).

Recently, the potential for analyzing co-occurrences of spatial events at higher order has been explored in Leibovici et al. (2008) and Leibovici (2010), in the context of correspondence analysis, the CAkOO method. This exploratory method breaks down the $\chi^{2}$ statistic for the lack of independence between the co-occurrences of the categorical variables in a similar way to that in which a principal component analysis breaks down the total variance (or inertia). One 
can call this independence a spatial independence since this is done using spatial contingencies: the co-occurrences. Multinomial distributions of spatial cooccurrences demand the consideration of other statistics or metrics which are linked to spatial patterns of multi-type marked event processes and multivariate point processes. As exploratory functions for structure and scale, entropy measures derived from these multinomial distributions of co-occurrences at a given order are considered.

We present here two approaches using spatial co-occurrence and the entropy measure. The first exploratory method considers all pre-selected variables and covariates of the events and analyses the plot of the observed spatial entropies from co-occurrences of order $k$ (a SOOk curve), along with its null hypothesis envelope (obtained here via Monte-Carlo simulation according to random labelling). The second method (in an approach similar to PEGASE) looks at the best clustering which can be obtained by hierarchical selection of covariates (SelSOOk method). SelSOOk can then provide an optimized selection of covariates to be used in a SOOk analysis or in a CAkOO analysis. CAkOO, SOOk and SelSOOk have been implemented in $\mathbf{R}$ (R Development Core Team, 2007) using packages for managing spatial data (see Bivand (2008) for a comprehensive state of the art) and in particular spatstat (Baddeley and Turner, 2005) and PTAk (Leibovici, 2010).

There are various contexts in environmental monitoring where the detection of changes in a complex system may involve the study of multi-covariate patterns, i.e. studying interactions, rather than marginal changes. These contexts include vegetation succession and the spread of disease, and two example datasets from these application domains are used to illustrate the methods derived for this purpose. The examples, which are discussed in detail below, are taken from an epidemiological study in England, and a vegetation biodiversity survey in Greece. 


\section{Environmental dataset examples}

For the purposes of this paper, the techniques outlined above and developed in further sections were tested on two point datasets with multivariate attributes. Both were projected to local National Grids for the areas from which they were collected, with distance units in meters. In both datasets, some elements of spatial structure had been identified in previous studies, using well-tested techniques. This meant that they were useful benchmarks on which to test these novel exploratory methods.

\subsection{Epidemiological dataset}

The first dataset is from an epidemiological study, which investigated the distributions of meticillin-resistant Staphylococcus aureus (MRSA) and its antibioticsensitive counterpart, (SA) in and around a British city (Bastin et al., 2007b). The variates recorded, i.e. the marks (labels), consisted of information on patient gender, age group, the date on which the disease was diagnosed, and the nature of the disease (MRSA or SA). The spatial locations of the point records are derived from postcode centroids, so that each patient is sited at the population-weighted center of the postcode polygon in which they reside. In the Bastin et al. (2007b) study, the data was subjected to kernel density filtering and spatial scan techniques. When compared to "null" Monte Carlo simulations which echoed the underlying population density and age structure, significant spatio-temporal clusters of MRSA were discovered using these techniques.

This data has a naturally patchy spatial structure, (see Figure 1), because of the underlying heterogeneity in population density, and the "snapping" of a patient's location to the postcode centroid, rather than their exact address, could potentially cause problems for studies where it is necessary to calculate real inter-point distances. However, for a random labelling approach this is less of an issue, since all sets of labelled points are subject to the same artificiallyimposed granularity. For this reason, a random labelling approach (MRSA/SA) was applied in Bastin et al. (2007b) to investigate whether MRSA cases tended to cluster together more strongly than SA cases; this was found to be the case 


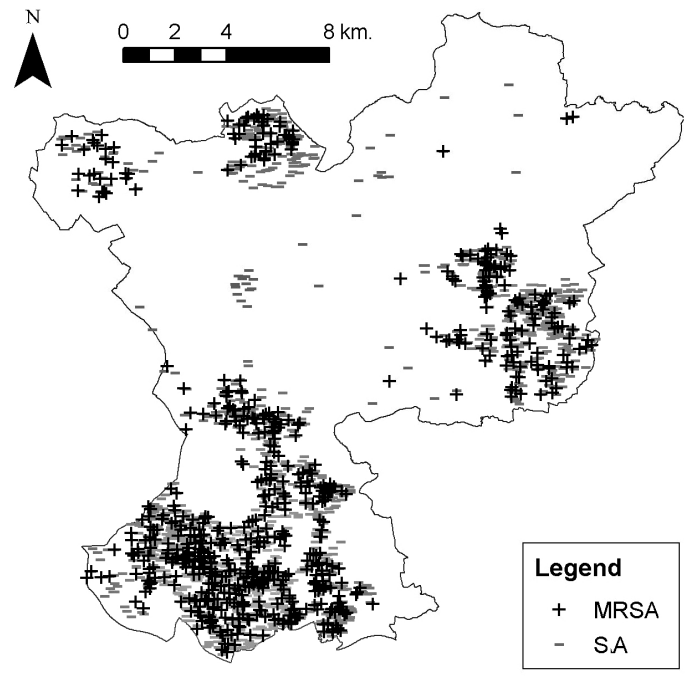

Figure 1: MRSA $(N=428)$ and SA $(N=1450)$ records for the epidemiological dataset.

only in patients over 65 . Though this analysis was stratified by age, in analysing co-occurrence and clustering it used only one mark, i.e. disease resistance to antibiotics. The methods demonstrated in this paper aim to extend the analysis to a multivariate approach; that is, to identify whether the spatial occurrences of the MRSA and SA marks, together with the age categories or other available risk factors, show spatial structures, clusters or a tendency to cluster.

\subsection{Plant community dataset}

The second dataset consists of 269 vegetation samples collected from an area of limestone phrygana near Thessaloniki, in Northern Greece (22.93N, 40.74 E: see Figure 2). Phrygana is a secondary vegetation community characterized by sclerophyllous dwarf-shrubs at varying densities, with areas of grassland and bare soil. The spatial sampling strategy was a stratified random plan where three transects were randomly placed within each of a number of regular grid squares. On each transect, the abundance and occurrence of numerous individual plant species was recorded, giving a detailed picture of the local plant community. This detailed plant community data was used to generate fuzzy memberships for each sample in four numerically-characterised vegetation cat- 
egories, identified using DECORANA (see Table 1) (Bastin et al., 2007a).

\begin{tabular}{l|l}
\hline Tclu1 & "Open Grassland" \\
Tclu2 & "Dense Cistus/Quercus scrub" \\
Tclu3 & "Medium Cistus scrub" \\
Tclu4 & "Dense Quercus/Pyrus/Pinus" \\
\hline
\end{tabular}

Table 1: Community characterization of the fuzzy clustering membership.

Although the defined communities represent complex assemblages of species, for simplicity's sake in Table 1 they have been named after their dominant, or defining, components. These assemblages tend to mix and grade into one another across the landscape, resulting in mixed memberships at some points, and "pure" samples at others.

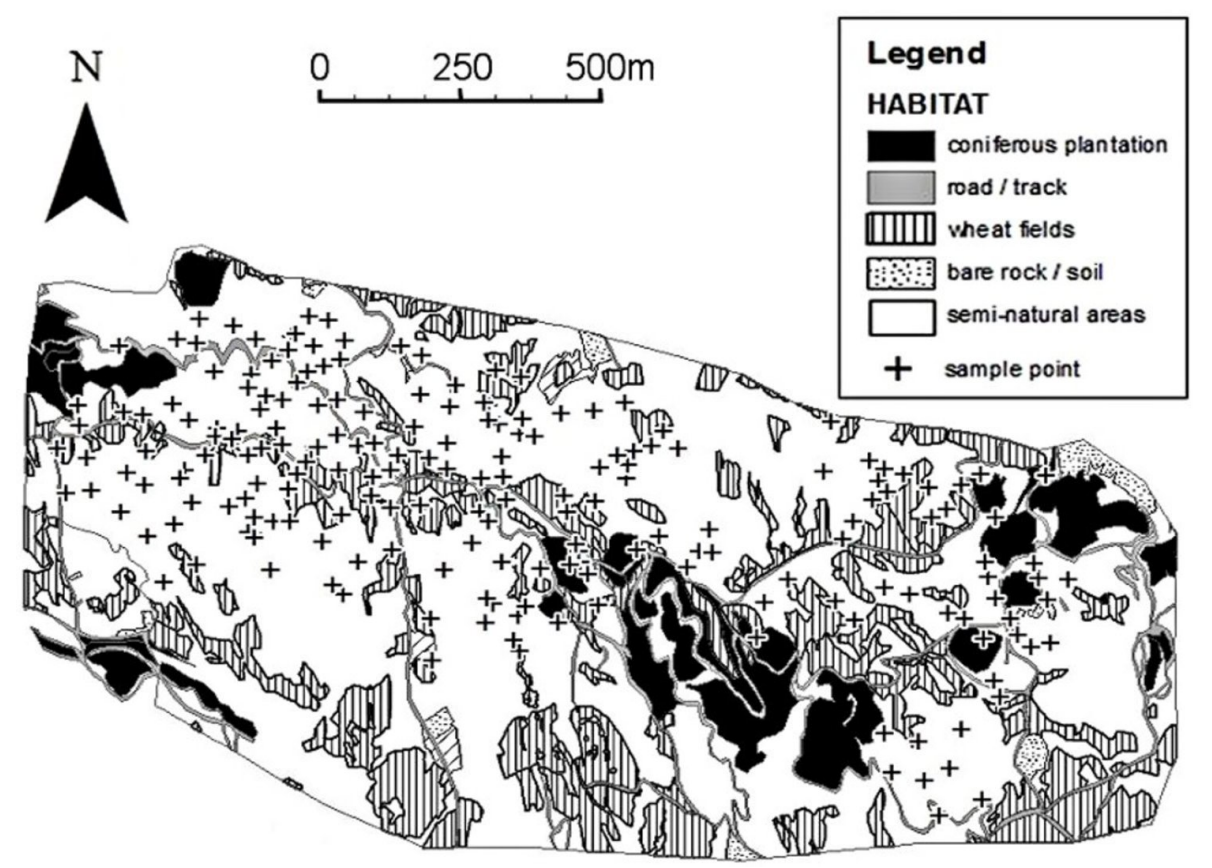

Figure 2: Locations of sampling points within the vegetation dataset

The aim of the original study was to see whether unknown sites could be easily classified into complex vegetation categories from simpler metrics which are more easily and cheaply measured, and which can often be assessed from remotely-sensed imagery. This can be crucial for monitoring purposes in avoid- 
ing long and expensive surveys. For this reason, a number of simpler variables were collected, as follows: patchiness of the transect (Patch) (i.e., variation in cover type); Texture of the transect (Tex) (i.e. total changes in vegetation height along its length); slope (Slope) and aspect (Aspect) of the sample site; $\%$ of area covered by open grassland/ground (open), low shrub (low), high

shrub (high), tree cover (tree), and mixed shrub and tree cover (s\&t) (bold face acronyms will be used in the analysis section). In the analysis described here, the focus is on identifying the best "simple" variables for predicting and describing the spatial distribution of the cluster membership values that represent the richer multi-species dataset.

\section{From $2^{\text {nd }}$-order to $k^{\text {th }}$-order analysis}

The events which are recorded spatially for a particular study in a geographicrelated discipline, e.g. ecology, epidemiology or crime studies, are usually considered in spatial statistics as being associated with a point process, that is "a stochastic mechanism generating a countable set of events"(Diggle, 2003; Bivand, 2008); locations of events correspond to points in the studied spatial domain. A spatial point pattern is thus seen as a realization of the point process $X$ in a bounded domain $W$, leading to a finite number of observations, each $x \subset X \cap W$. When attribute values are attached to each location or each event, one gets a marked point process: that is, a realization of a point process and a marking process. Each point $x$ has a mark $m$ and the observation is $x_{m}$. The challenge for point pattern analysis is to assess the associations of marks for the same process or for different processes, and the degree to which they depend on other covariates. The cross- $K$ function can be used, but becomes limited when studying multivariate systems as the number of comparisons of pairs can increase dramatically.

\subsection{Extending 2nd-order statistics}

Practical questions often deal directly with higher-order statistical issues. For example, an ecologist may be interested to know whether, within a given 
landscape, the association of two specific plant species occurs more often on one specific soil type. A more exploratory approach would be to assess whether there is a particular structuring of plant species and soil types with habitat types. For this purpose, quadrat count distributions associated with sampling designs have been used intensively in landscape ecology but suffer from the MAUP (Modifiable Area Unit Problem) issue, as discussed in Wagner and Fortin (2005). Quadrat count statistics are also often first-order summaries, targeting, for example, a richness index. The principle of quadrat counts shows, nonetheless, that the focus is on co-occurrences of events and the distribution of these cooccurrences.

Classical estimators of statistics based on moments are in fact expressed by co-occurrences. For example, for an inhomogeneous marked point process, a symmetric cross-type Ripley's $K$ statistic can be expressed:

$$
\hat{K}^{S}{ }_{l m}(d)=\frac{1}{|W|} \sum_{\left\{x_{l}, x_{m}\right\} \subset X \cap W} \frac{1\left(d\left(x_{l}, x_{m}\right) \leq d\right)}{\lambda\left(x_{l}\right) \lambda\left(x_{m}\right)}
$$

where $|W|$ is the area of the window $W$ over which the marked point process $X$ is evaluated; $l$ and $m$ are the two marks of interest; $x_{l}$ and $x_{m}$ are any two observed points, respectively with marks $l$ and $m$, of the pattern associated with $X$; the sum is over pairs of points, sampled from $X$ with marks $l$ and $m$, and found in $W ; d$ is the distance of co-occurrence and the function 1() is the indicator function (its value is 1 if the evaluated expression is true, 0 otherwise); and $\lambda\left(x_{m}\right)$ is the intensity of the process at point $x_{m}$. The above formula is adapted from Baddeley et al. (2000) without edge correction.

A simple extension of Ripley's $K$ Equation (1) to higher orders gives, here choosing the order $k=3$ :

$$
\begin{gathered}
\hat{K}^{S}{ }_{\text {mop }}(d)= \\
\frac{1}{|W|} \sum_{\left\{x_{m}, x_{o}, x_{p}\right\} \subset X \cap W} \frac{1\left(d_{P}\left(x_{m}, x_{o}, x_{p}\right) \leq d\right)}{\lambda\left(x_{m}\right) \lambda\left(x_{o}\right) \lambda\left(x_{p}\right)}
\end{gathered}
$$

where $d_{P}$ is the maximum distance between any pair of the evaluated points (the distance used in the examples is the Euclidean distance). The capital subscript 
$P$ refers to this Pairwise consideration of distance, and is distinct from the lower-case subscript $p$ used to denote a mark. When the indicator function $1\left(d_{P}\left(x_{m}, x_{o}, x_{p}\right) \leq d\right)$ equals 1 , a co-occurrence of order 3 between marks $m$, $o$, and $p$ for the given points is counted. If the denominator is constant the numerator of the statistic counts the co-occurrences between these marks. The extension for any order $k>3$ is a straightforward extension of Equation (2). The statistic of Equation (2) accounts for lower-order moments as well. For example, if, in Equation (2) the marks $m$ and $o$ are the same, then, the numerator of the statistic accounts for $2^{r d}$-order co-occurrences as well as $3^{\text {th }}$-order of the marks $m$, and $p$. To be purely of $k$ th-order a constraint must be added, as follows: for equal marks, the corresponding points have to be different.

\section{Multiple Co-occurrences for Spatial Entropy}

If, conditionally to the marks, the process is assumed to be homogeneous, then the intensities can be estimated by $\hat{\lambda}\left(x_{l}\right)=\hat{\lambda}_{l}=n_{l} /|W|$, where $n_{l}$ is the number of points with mark $l$. In this case, equations such as (1) and (2) correspond to a ratio representing lack of independence, as in the $\chi^{2}$ measure of independence. For example, the $3^{r d}$-order statistic can be written:

$$
\begin{gathered}
1 /|W| \sum_{x_{l}, x_{m}, x_{p} \subset X \cap W} \frac{1\left(d_{P}\left(x_{l}, x_{m}, x_{p}\right) \leq d\right)}{\lambda\left(x_{l}\right) \lambda\left(x_{m}\right) \lambda\left(x_{p}\right)}= \\
\frac{1 /|W| \sum_{\{\}} 1\left(d_{P}\left(x_{l}, x_{m}, x_{p}\right) \leq d\right)}{\left(n_{l} /|W|\right)\left(n_{m} /|W|\right)\left(n_{p} /|W|\right)}= \\
\frac{1 /|W| \frac{n_{l m p}}{n_{\ldots}} \frac{n_{. .}}{n^{3}}}{\left(n_{l} /|W|\right)\left(n_{m} /|W|\right)\left(n_{p} /|W|\right) / n^{3}}=\frac{p_{l m p / \text { coll }} p_{\text {coll }}}{p_{l} p_{m} p_{p}} \\
= \\
O_{l m p} / E_{l m p}
\end{gathered}
$$

where $O_{l m p}$ is the observed probability of co-occurrences of three points with marks $l, m$, and $p ; E_{l m p}$ is the estimate of this probability under the hypothesis of complete independence of the collocating events. These probabilities have to be understood as normalized to the unit surface (because of $|W|$ ). Other notations and necessary derivations are: $n_{l m p}=\sum_{\{\}} 1\left(d_{P}\left(x_{l}, x_{m}, x_{p}\right) \leq d\right)$ 
is the number of collocations of the marks $l, m$ and $p ; n_{\ldots}=\sum_{l, m, p} n_{l m p}$ is the total number of $3^{r d}$-order collocations; $p_{l}=(1 /|W|) n_{l} / n$ is the probability of finding a point with mark $l$ in $\mathrm{W}, n$ being the total number of points.; $p_{\text {coll }}=(1 /|W|) n_{\ldots} / n^{3}$ is the probability of a triple of marks to collocate in $\mathrm{W}$ and $p_{l m p / c o l l}=n_{l m p} / n_{\ldots}$ is the probability of finding the marks $l, m$ and $p$ conditionally to have three co-occurrent points in $\mathrm{W}$.

Without making any homogeneity assumption on the sub-processes ${ }^{1}$, Leibovici et al. (2008) and Leibovici (2010) describe the analysis of a contingency table of co-occurrences, such as the multiway table $n_{l m p}$ of Equation (3), using correspondence analysis for multiway table: the CAkOO method (Correspondence Analysis of k cO-Occurrences). CAkOO analyses spatial independence but does not directly test or quantify the existence of a pattern, nor does it test at which "scale" it exists (one distance $d$ of collocation is chosen in advance). A multiscale analysis can nonetheless be done by adding a scale dimension in the multiway table (Leibovici et al., 2007; Leibovici and Jackson, 2008).

Multivariate co-occurrence distributions, derived from one or different processes, describe the spatial structure or organization of the events and the interactions between or within the processes. Here are some examples of multivariate co-occurrence distribution choices using the plant community dataset; they are hypothetical and we used a very different analysis strategy with this dataset (see further with the SelSOOk analysis):

- A multivariate co-occurrence of interest would be the association between the clustering of the species communities (Tclu1-Tclu4), and the variables Patch (recoded into 3 categories) and Slope (recoded into 2 categories). Note that for this example, the point processes for all the variables are the same, so that one has a multivariate multivariable marking process on a single point process. For a given distance of co-occurrence, the collocation counts for the three variables are $n_{t p s}$, for collocations of points with a

\footnotetext{
${ }^{1}$ as in the rest of the paper.
} 
cluster membership $t$, a patchiness category $p$ and a slope category $s$, resulting in a table of dimension $4 \times 3 \times 2$.

- One can look at $n_{t_{1} t_{2} t_{3}}$, where only the structure of collocations of the variable identifying species cluster membership is of interest (only one process is in fact analyzed), giving a table of collocation counts of dimension $4 \times 4 \times 4$.

- Alternatively, one can look at $n_{i j k}$ where $i, j$ and $k$ are either a species cluster membership, a patchiness category and a slope category, so building a symmetric table of dimensions $(4+3+2) \times(4+3+2) \times(4+3+2)$, where all collocation patterns of order 3 are recorded.

In order to summarize the "meaningfulness" of a pattern, we need some index which can numerically compare structured to random configurations, i.e. a measure of "spatial entropy". Shannon entropy, originating in information theory, has commonly been used for this purpose on spatial data consisting of raw category distributions, and has also been adapted to take into account the adjacency counts of categories (O'Neill et al., 1988). Higher-order co-occurrence can be seen as an extension of adjacency. Using the multivariate co-occurrence distribution, one can assess the spatial structure and dependence of one (or more) multi-type marked process(es), using as spatial entropy the index:

$$
H_{S u}\left(C_{o o}, d\right)=-1 / \log \left(N_{C_{o o}}\right) \sum_{c_{o o}} p_{c_{o o}, d} \log \left(p_{c_{o o}, d}\right)
$$

where $C_{o o}$ is a multivariate co-occurrence of attributes considered from one or more multi-type marked point processes, generating the collocation counts with the multi-index $c_{o o}$, and thus the distribution $p_{c_{o o}, d}$. The collocation is for a chosen distance value $d$ and a chosen order $k$ - in most of the examples, $k=3$. The multi-index $c_{o o}$ has $k$ positions with values for each position depending on the marks involved (see the above examples). $N_{C_{o o}}$ is the number of multiindexes coo, that is the number of cells of the multiway table, so that the term $\log \left(N_{C_{o o}}\right)$ corresponds to a normalization relative to the entropy of a uniform 
distribution. $H_{S u}$ stands for spatial entropy relative to uniformity: the Shannon entropy is usually termed $H$ and the subscript ${ }_{S}$ is for spatial, ${ }_{u}$ to express the ratio to uniformity.

A univariate index can also be derived, by only considering as interesting values in $C_{o o}$ the co-occurrences of each category with itself, that is the ones corresponding to the hyper-diagonal of the co-occurrence table, a $k$ entries table, e.g. $n_{i i i}$ for an order $k=3$ :

$$
H_{s S u}\left(C_{o o I}, d\right)=-1 / \log (I) \sum_{i}^{I} p_{s(i), d} \log \left(p_{s(i), d}\right)
$$

with a given order of co-occurrences, defined by the length of the multi-index $s()$ and where $I$ is the number of categories. The self multi-index $s()$ expresses the fact that the observed probabilities are computed only using the hyper-diagonal of the counts of co-occurrences, e.g. with co-occurrences of order 3 :

$$
p_{s(i), d}=n_{i i i} / \sum_{i} n_{i i i}
$$

The self spatial entropy, also normalized to uniformity, $H_{s S u}$, then measures the spatial pattern according to clustering of occurrences of the same categories (derived from attribute values) of the points. As for the classical Shannon entropy, low values of $H_{S u}$ and/or $H_{s S u}$ means the existence of some structure in the distribution, which in this case implies a spatial structure of the marks. Since the spatial measures are normalized, as they approach 1, so the distribution of co-occurrences gets closer to the uniform distribution.

\section{Examples of SOOk and SelSOOk analyses}

For the SOOk method, plotting the spatial entropy against distance of cooccurrence allows the study of potential patterns at different scales. Evidence of low entropy, due to spatial configuration of the marks when compared to the same point process with different marks, can be obtained from Monte Carlo test simulations using random labelling. 


\subsection{Lansing woods dataset}

Before looking at the example datasets described at the beginning of this paper, we looked at a classical point pattern data existing in the literature and in the R-package spatstat (Baddeley and Turner, 2005): the Lansing data, consisting of 2251 trees in a $924 \times 924$ feet plot rescaled to a unit square. The

order 3 collocations for the 6 classes: blackoak hickory maple misc redoak whiteoak

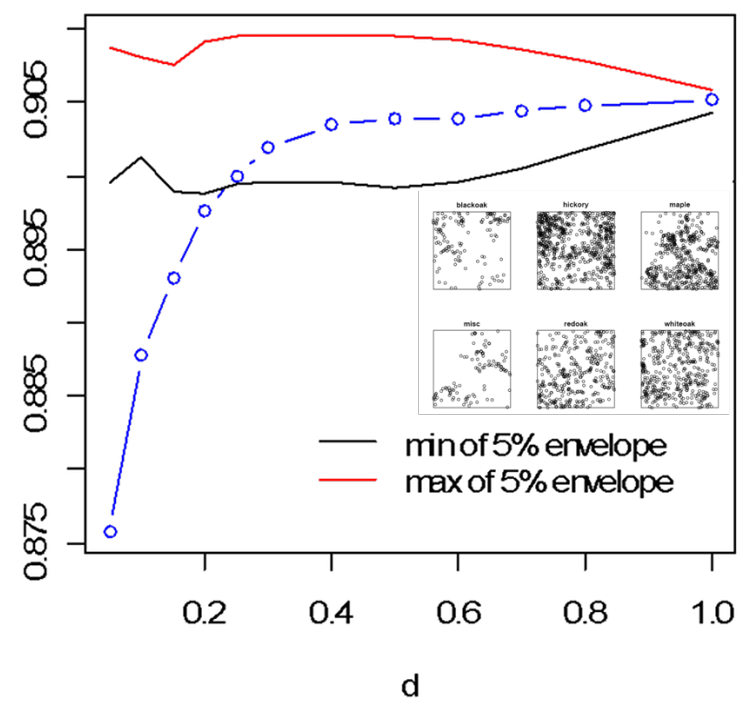

Figure 3: Scale effect of $3^{r} d$-order spatial entropy, $H_{S u}$, with a $5 \%$ envelope from random permutations, on the Lansing data (right panel); included panels are the processes for each class category (in the given order of marks and by row)

tree marks of the Lansing woods data show some structure up to 0.2 units, as the spatial entropy has low values outside the envelope computed under the null hypothesis of random labelling (see Figure 3). For the low spatial entropy at $d=0.15$, we described in Table 2 the spatial structuring of the trees by looking at each term in the sum which constitutes $H_{S u}$. Marginals, i.e. sums of the terms involving one particular category, indicate that blackoak and misc categories contribute to a low entropy, so are structuring categories. Not surprisingly their sub-processes are visually the least homogeneous (sub panels of Figure 3). Then the relatively lower percentage for redoak against 
the rest appears to have a structuring effect. Looking at elementary terms of the spatial entropy (lower part of Table 2), the most structuring are in clusters of misc and blackoak trees with misc trees (mimibl) or with blackoak trees (miblbl). The notation (mihibl) means a collocation of order three for the trees $m i, h i, b l$ corresponding to the two first letters of the tree species. The least structuring appear to be collocations of hickory trees (hihihi), and of maple trees (mamama), and both species represent marginally (27\%) and (23\% respectively. Nonetheless one must be aware that $-p \log (p)$ can be very small for very small or very high $p$ 's, so a highly structuring event may be very rare or very frequent: $p_{\text {mimibl }}=0.0002$ compared to the maximum of probability of collocation of order $3, p_{\text {hihihi }}=0.16$ which must be compared to the probability under uniformity for 56 types of collocations, $1 / 56 \approx 0.02$.

This data has been used to illustrate $2^{\text {nd }}$-order spatial point pattern analysis in the literature (Diggle, 2003). A direct comparison of the methods is difficult as Ripley's $K$ can at most use two different marks. Some comparison can be made using the self-spatial entropy $\left(H_{S S u}\right)$ as it is a direct extension of $K$ from 2 to $k>2$ co-occurrences. The self spatial entropy is $H_{s H u}=0.62$ and the probability of self-collocations of order 3 are also given in Table 2. So as $H_{s S u}<$

\begin{tabular}{l|c|c|c|c|c|c}
\hline Marginals & blackoak & hickory & maple & misc & redoak & whiteoak \\
\hline of $H_{S u}=0.893$ & $7 \%$ & $27 \%$ & $23 \%$ & $7 \%$ & $16 \%$ & $20 \%$ \\
$n_{i i i} / \sum_{i} n_{i i i}$ & 0.008 & 0.550 & 0.301 & 0.004 & 0.043 & 0.094 \\
$p_{i .}$ & 0.056 & 0.303 & 0.244 & 0.050 & 0.150 & 0.197 \\
\hline \hline parts of $H_{S u}$ & & mi mi bl & mi bl bl & re mi bl & wh mi bl & mi ma bl \\
5 lowest values & & $0.0152 \%$ & $0.0180 \%$ & $0.0365 \%$ & $0.0467 \%$ & $0.0510 \%$ \\
\hline & & hi hi hi & ma ma ma & wh hi hi & wh ma ma & ma hi ma \\
5 highest values & & $3.38 \%$ & $2.23 \%$ & $1.70 \%$ & $1.41 \%$ & $1.35 \%$ \\
\hline \hline
\end{tabular}

Table 2: Decomposition analysis of $H_{S u}$ at collocation distance $d=0.15$

$H_{S u}$, more self structuring of the different tree species is observed than "cross" structuring, with hickories and maple trees aggregating a lot more relatively to the other species, as was also found in Diggle (2003). Diggle also found a segregation effect between maple and hickory trees. This can be identified by the fact that for all other pairs of tree species, $H_{s S u}<H_{S u}$ (where $C_{O O}$ uses only the two tree species), while for maple and hickory trees the reverse was 
observed, expressing rarer cross-collocations.

\subsection{MRSA dataset}

For the epidemiological survey (MRSA data, see Section 2.1), the MRSA status + (resistant $)$ and - (sensitive), of the 1878 patients, were combined with their age code categories 1 ( $<45$ years), 3 ( $45-75$ years), 5 ( $>75$ years), to generate the six distinct marks of the process to analyze using the SOOk approach; Figure 4 displays the sub-patterns for each mark. The top panel of
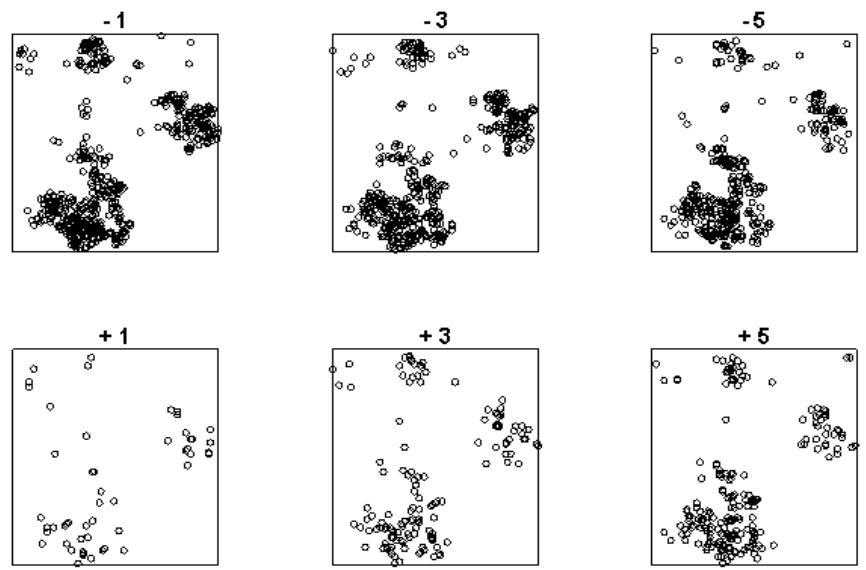

Figure 4: MRSA data, split patterns for each mark combining MRSA status and age categories (see text).

Figure 5 demonstrates no evidence of spatial structure for this distribution. Two local minima at $2000 \mathrm{~m}$ and $7400 \mathrm{~m}$ suggest some effects which are, however, due only to the geographical spread of the sample, i.e., its underlying, heterogeneous, population density in the form of three conurbations. A division of the area into three zones representing these conurbations, and subsequent computation of the envelope by allowing re-sampling only within each of the three zones, did not improve the result. Localizing the $H_{S u}$ entropy at $d=2000 \mathrm{~m}$ did demonstrate some tendency towards clustering, (see grey panel of the top plot in Figure 5), which was found to be linked to age in Bastin et al. (2007b) where the association of age and MRSA status appears selective. The self spatial entropy may indeed provide the same evidence - for an illustration, see the lower panel of 
Figure 5, where self collocations of order 3 for age categories are also displayed (at $d=2000 m$ ) for the sub-processes defined by the MRSA status. There is clearly a structure of age within patients with MRSA as compared to a nearly uniform distribution for the SA status, and this is confirmed by the histograms shown at $d=2000 \mathrm{~m}$ (bottom of Figure 5). One benefit of the method presented here is that all 'age' marks could be considered simultaneously, and their relative structuring effects compared, while in the original study, three separate analyses were required to identify the same phenomenon - one for each age group.

\subsection{With the plant community dataset}

The spatial entropy measure based on co-occurrences can also be used for regression tree clustering. An approach similar to PEGASE (Phipps, 1981) is taken here. SelSOOk can be performed either with no target variable or with a target variable.

With no target variable, a hierarchy of variables is looked for according to the best spatial structure obtained at each level of the hierarchy. A spatial structure is defined by the spatial pattern of the marking of the points using a given series of variables. At each level of the hierarchy, the set of all preceding variables in the hierarchy constitutes the series of variables to be used. The optimization for best spatial structure is obtained by looking for the minimum joint spatial entropy ${ }^{2}$ of the set of variables: at the $i^{\text {th }}$ step, the variable $X_{(i)}$ minimizing $H_{S u}\left(X_{(1)}, X_{(2)}, \ldots, X_{(i)}\right)$ is added into the hierarchy.

With a target variable, the best hierarchy of variables is obtained as in a stepwise regression. Note that here the focus is not on the best spatial structuring set of variables but the best at explaining the spatial structure of the target variable. At each step, the set of variables acts like regressors and the conditional entropy ${ }^{2}$ is the criterion to minimize. Joint and conditional entropies were derived from Equation (4) and using classical entropy formula ${ }^{2}$

\footnotetext{
${ }^{2}$ The joint entropy of two variables $X$ and $Y$ is the $H(X, Y)$, the entropy of joint probability distribution; the conditional entropy, $H(Y \mid X)$ is the expectation of the entropies on the conditional probabilities given the values of $X$; the joint entropy and the conditional entropy are linked by: $H(Y \mid X)=H(X, Y)-H(X)$, see for example Reza (1994).
} 

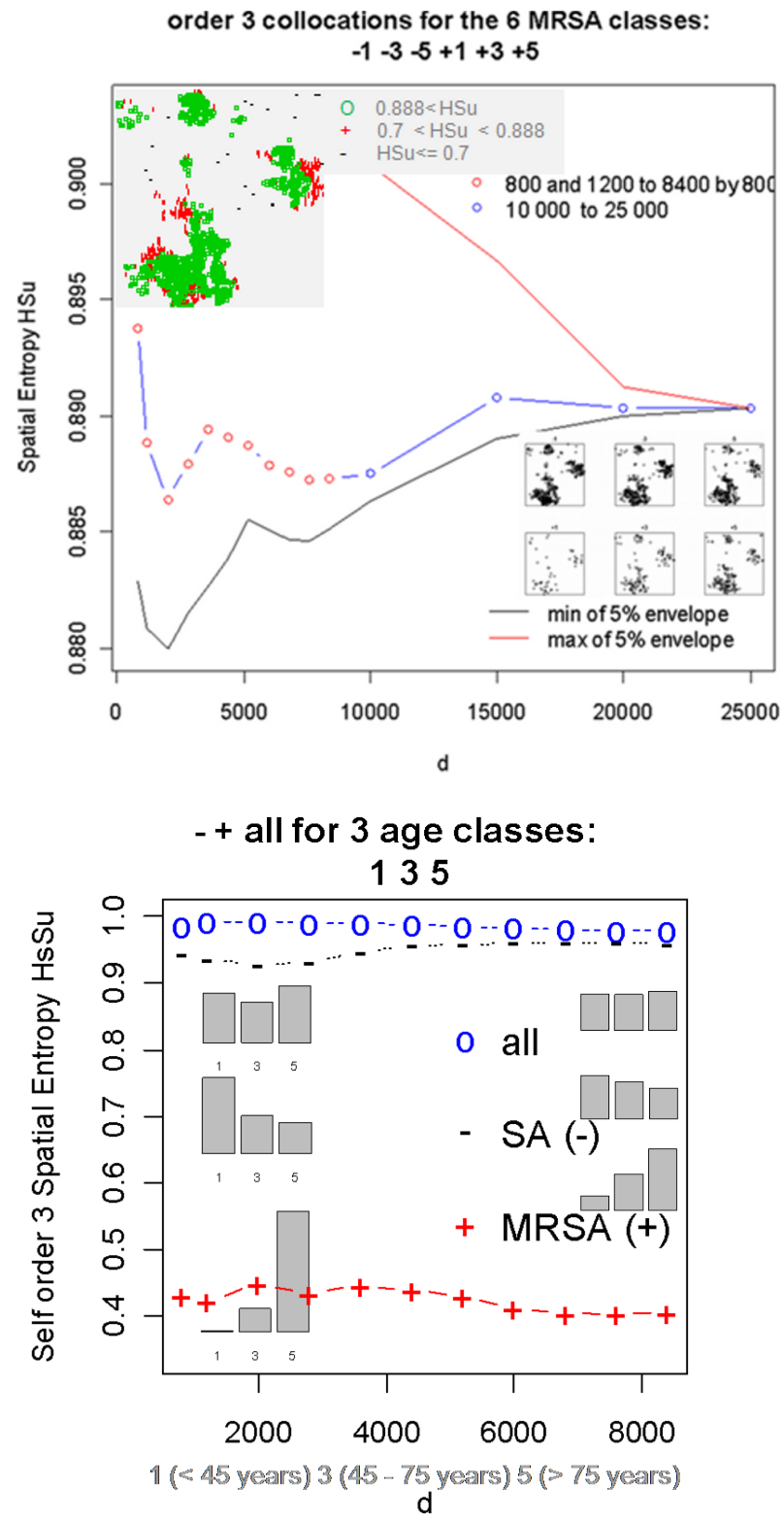

Figure 5: Scale effect of $3^{\text {rd }}$-order spatial entropy on the MRSA data: (top panel) on the combined age and MRSA status 6 classes with a $5 \%$ envelope from random permutations, the grey panel displays a localized $H_{S u}$ at $d=2000 \mathrm{~m}$ split in three classes; (bottom panel) comparison of the self-spatial entropy for the 3 age classes for the whole process and the sub processes for each MRSA status; included panels are the age histograms for each MRSA category (by row): histograms based on self-collocation counts (at $d=2000 \mathrm{~m}$ ) on the left and simple histograms of the categories on the right. 
(the normalization to uniformity cannot be used for the conditional entropy). For simplicity of implementation the criterion minimized for the $i^{t h}$ variable was: $H_{S}\left(Y, X_{(1)}, X_{(2)}, \ldots, X_{(i-1)} \mid X_{(i)}\right)$ instead of $H_{S}\left(Y \mid X_{(1)}, X_{(2)}, \ldots, X_{(i-1)}, X_{(i)}\right)$. These two optimization are very similar, as:

$$
H\left(Y \mid X_{(1)}, X_{(2)}\right)=H\left(Y, X_{(1)} \mid X_{(2)}\right)-H\left(X_{(1)} \mid X_{(2)}\right)
$$

so minimizing the left hand of equation (for best $2^{\text {nd }}$ variable) would also ensure that the additional variable $X_{(2)}$ is the least "correlated" with the previous variable $X_{(1)}$.

True tree clustering proceeds by splitting the data at each stage where a new clustering variable is selected, leading to classes which are not necessarily built with the same set of variables. Only the procedure without split has been implemented here. Note that there is still a tree, and that this tree is symmetrical: at each level, the nodes are split with the same variable and the optimization is done per level, not per node (on each split sample) as in a true tree clustering.

The plant community dataset (see Section 2.2), containing 269 point samples with attributes representing vegetation metrics, was used to illustrate the alternative SelSOOk analysis. On Figure 6 the analyses are: (i) a tree clustering of the vegetation indices as they capture the spatial structure (ii) and then as they explain the spatial structure of the crisp clustering membership variable $\mathbf{T c l u}$ (variable coded from allocating each sample to the cluster in which it has highest membership). For simplicity, the vegetation indices were here coded as binary: high and low values based on their histograms. The sequence of the best four variables for joint spatial entropy is identical (tree, low, Patch, open) at all distances of collocation. However, the fifth best variable depends on collocation distance: in most cases it is $\mathbf{s} \& \mathbf{t}$ but at 5 meters (the finest spatial scale) it is high.

As expected, the joint spatial entropy increases as variables are added to describe the structure of the data (top panel of Figure 6). Tree cover (tree) is persistent at all scale and well in front of the other variables. The sequence 


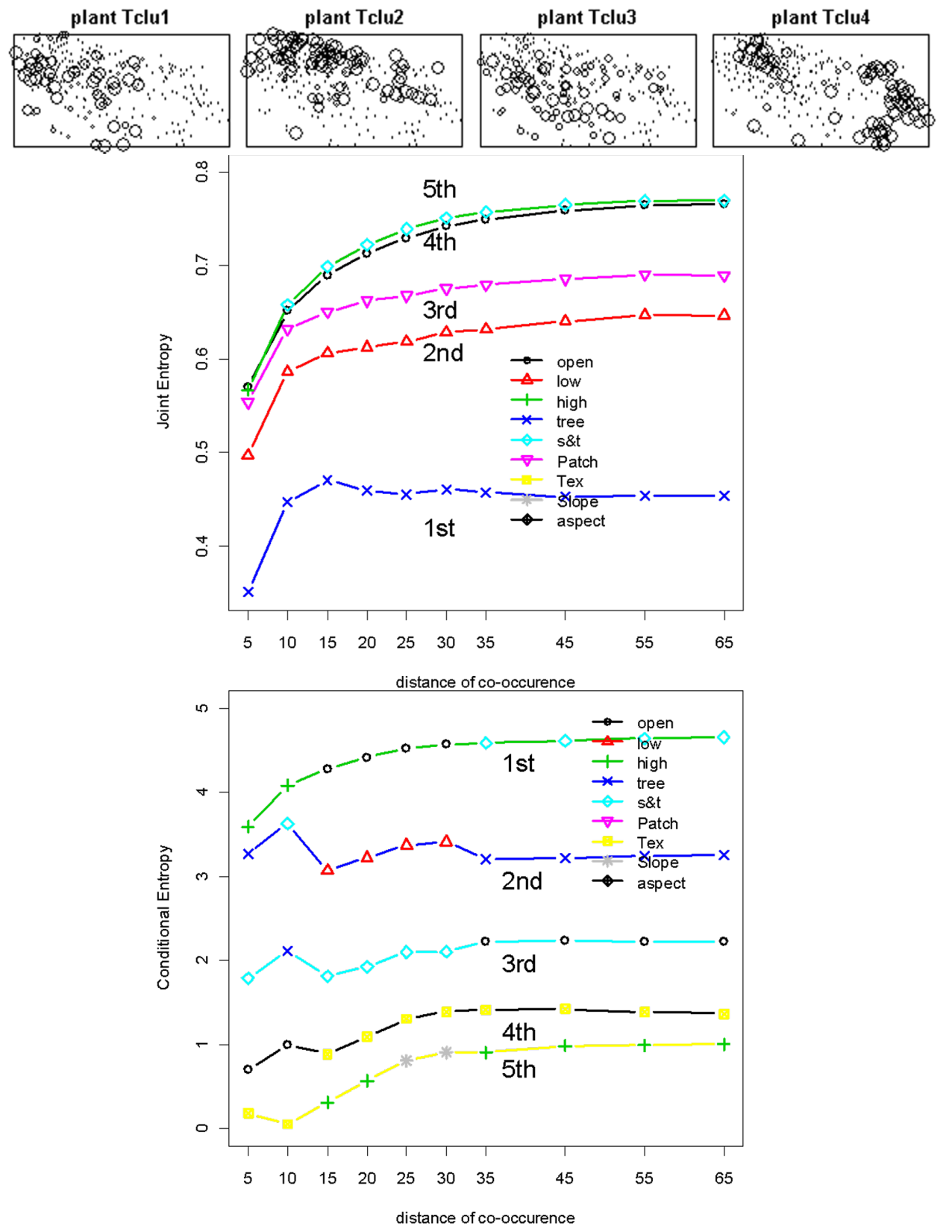

Figure 6: Scale effect with $3^{\text {rd }}$-order SelSOOk tree clustering of the plant community dataset; [Top] subsequent values $H_{S u}\left(X_{(1)}, X_{(2)}, \ldots, X_{(i)}\right)$ where $i=1, \ldots, 5$ is the best $i^{t h}$ selected variable. [Bottom] same with the conditional entropy $H_{S}\left(Y, X_{(1)}, X_{(2)}, \ldots, X_{(i-1)} \mid X_{(i)}\right)$, where $Y$ is the crisp classes from transect data clustering (top panel), and $X_{(i)}$ 's are the vegetation coded indices. 
of the variables is persistent through the change in scale factor (the distance of collocation). Note that the relief variables, Slope and Aspect, do not play an important part here.

On the other hand, the other SelSOOk analysis (in the lower panel of Figure 6), showed some effect from Slope but as fifth variable in the hierarchy and only at 25 and 30 meters of collocation. The decrease of the conditional entropy as the hierarchy of variables expands to explain the crisp clustering target variable was also expected from the classical formula of entropy. Explaining the crisp clustering coming from transect data (Tclu), one must notice the change of hierarchy as the scale factor changes, with an approximate consistency for three scale divisions: micro (5m-10m), medium (15m-30m), larger (35m-65m). The variables selected in the sequences show some differences to the ones obtained for (i), as follows:

- 05-10 meters: high, tree, s\&t, open, Tex

- 10-10 meters: high, tree, open, s\&t, Tex

- 15-20 meters: open, low, s\&t, Tex, high

- 25-30 meters: open, low, s\&t, Tex, Slope

- 35-65 meters: s\&t, tree, open, Tex, high.

At finer scale high shrubs comes in first to explain the plant community clustering variable, but was not in the first four variables for the analysis in (i). Still comparing to the results for (i), Patchiness disappears from the best four variables, as not linked to the spatial distribution of the plant community clustering variable. These results are consistent with the observed patterns, and give valuable further insights. When the four clusters are referenced back to the vegetation communities they represent (Table 1), it can be seen that the "Open" (Tclu1) and "Medium Shrub" (Tclu3) clusters are widely and evenly spaced across the landscape, reflecting the way in which variables s\&t and open move up the hierarchy with increasing spatial lag. In contrast, dense shrub and 
tree communities are characteristically highly spatially clustered, leading to the predominance of variables high and tree at the smallest landscape grain. Tex, which represents the local variability in structure at each sample point, is important at every spatial scale but never dominates as the driver in spatial structure.

\section{Discussions and Further issues}

Considering multiple co-occurrences' distributions for one or more point processes, a generic spatial entropy index was derived to analyze dependence in marked point processes at different scales (collocation distances). SOOk and SelSOOk methods were developed, which;

- analyze higher-order statistics towards clustered occurrences versus uniformity;

- generate tree classification of variables with an optimization either looking for minimum joint spatial entropy, or minimum conditional spatial entropy when a target or dependent variable is to be explained.

The choice of the order of co-occurrence plays an important role. Other experiments suggest that, for one categorical variable (one set of marks), increasing the order increases the discriminatory power offered by the spatial entropy index, between spatially-structured data versus less structured or randomly-patterned data (Leibovici, 2009). In these experiments, we used the $k$-values 2,3 and 4 . Obviously, increasing the order puts into question the necessary size of the studied sample for the underlying estimation of high order moments. A downside of the methods is about interpreting the results, particularly with the SOOk method where detecting a tendency to clustering with a pooled index does not inform which profiles (out of all the categories involved) are responsible for this effect. The CAkOO method may help in this description. To assess the significance of the observed scale effects for the spatial entropy index, we checked one of the possible hypotheses, namely the random labelling hypothesis which is conditional on the locations. When different point processes express different marks, one might also wish to test their independence (van Lieshout and 
Baddeley, 1999). Note that here, as $H_{S u}$ and $H_{s S u}$ are relative to uniformity of the co-occurrences, they measure something to be related to the spatial independence of the sub-processes. However, a theoretical variance is needed to be able to provide an confidence envelope under this hypothesis. We did not investigate the usual independence test, considered in point pattern analysis, of complete spatial randomness where the hypothesis is that the point pattern comes from an homogeneous Poisson process. This hypothesis will, however, be a useful starting point for further investigations..

A Ripley's $K$ extension, (see Equation 2), has been introduced here primarily as a conceptual link with established point pattern analysis, but as a generalization of a well known statistic in point pattern analysis, this extension is interesting in its own right; future work on its stochastic properties, and specifically its sampling distribution, will create opportunities for further hypothesis testing. The relationship to existing methods of exploratory point pattern analysis has been partially investigated in this paper by comparison to previous analyses, and some specific benefits and limitations introduced by the higher-order approach have been identified. These will be further investigated in order to identify specific contexts for which this extension and its derived methods are particularly suited, and to identify data, applications and sample sizes where robust and meaningful results can be achieved.

\section{References}

Baddeley, A., Møller, J., Waagepetersen, R., 2000. Non- and semi-parametric estimation of interaction in inhomogeneous point patterns. Statistica Neerlandica 54 (3), 329-350.

Baddeley, A., Turner, R., 2005. spatstat: An R package for analyzing spatial point patterns. Journal of Statistical Software 12 (6), 1-42.

Baddeley, A., Gregori, P., Mateu, J., Stoica, R., and Stoyan, D., 2006. Case Studies in Spatial Point Pattern Modelling. Springer-Verlag, New-York Inc, 306pp. 
Bastin, L., Fisher, P., Bacon, M., Arnot, C., Hughes, M., 2007a. Reliability of vegetation community information derived using DECORANA ordination and fuzzy c-means clustering. In: Kokhan, A. M. . S. (Ed.), Geographic Uncertainty in Environmental Security. Springer, pp. 53-74.

Bastin, L., Rollason, J., Hilton, A., Pillay, D., Corcoran, C., Elgy, J., Lambert, P., De, P., Worthington, T., Burrows, K., 2007b. Spatial aspects of MRSA epidemiology: a case study using stochastic simulation, kernel estimation and SaTScan. International Journal of Geographical Information Science 21 (7), 811-836.

Bel, L., Allard, D., Laurent, J., Cheddadi, R., andBar Hen, A., 2009. CART algorithm for spatial data: Application to environmental and ecological data. Computational Statistics \& Data Analysis 53, 3082-3093.

Bivand, R., 2008. Applied Spatial Data Analysis with R, 1st Edition. SpringerVerlag, New York Inc, 374pp.

Breiman, L., Friedman, J., Olshen, R., Stone, C., 1984. Classification and regression trees. Wadsworth statistics/probability series, Wadsworth International Group, Belmont, CA, 358pp.

Diggle, P., 2003. Statistical Analysis of Spatial Point Patterns, 2nd Edition. Hodder Arnold, London, 159pp.

Diggle, P., Gomez-Rubio, V. Brown, P., Chetwynd, A., Gooding, S., 2007. Second-order analysis of inhomogeneous spatial point processes using casecontrol data. Biometrics 63, 550-557.

Leibovici, D., 2010. Spatio-temporal multiway decomposition using principal tensor analysis on k-modes: the R package PTAk. Journal of Statistical Software $34(10), 1-34$.

Leibovici, D., 2009. Defining spatial entropy from multivariate distributions of co-occurrences. Spatial Information Theory 2009, Published in: Lecture Notes in Computer Science, vol. 5756/2009, 392-404. 
Leibovici, D., Bastin, L., Jackson, M., 2008. Discovering spatially multiway collocations. In: GISRUK Conference 2008, Manchester, UK, 2-4 April, 2008. pp. $66-71$.

Leibovici, D., Jackson, M., 2008. Multiscale integration for Spatio-Temporal ecoclimatic ecoregioning delineation. In: Geoscience and Remote Sensing Symposium, 2008. IGARSS 2008. IEEE International. Vol. 3. pp. III - 996-III 999.

Leibovici, D., Quillevere, G., Desconnets, J.-C., 2007. A Method to Classify Ecoclimatic Arid and Semi-Arid Zones in Circum-Saharan Africa Using Monthly Dynamics of Multiple Indicators. IEEE Transactions on Geoscience and Remote Sensing 45 (12), 4000-4007.

Li, X., Claramunt, C., 2006. A spatial entropy-based decision tree for classification of geographical information. Transactions in GIS 10 (3), 451-467.

Lotwick, H., Silverman, B. W., 1982. Methods for analysing spatial processes of several types of points. Journal of Royal Statistical Society B (44), 406-413.

O’Neill, R., Krummel, J., Gardner, R., Sugihara, G., Jackson, B., DeAngelis, D., Milne, B., Turner, M., Zygmunt, B., Christensen, S., Dale, V., Graham, R., 1988. Indices of landscape pattern. Landscape Ecology 1 (3), 153-162.

Phipps, M., 1981. Entropy and community pattern analysis. Journal of Theoretical Biology 93 (1), 253-273.

Quinlan, J., 1986. Induction on decision trees. Machine Learning 1, 81-106.

R Development Core Team, 2007. R: A Language and Environment for Statistical Computing. Vienna, Austria, ISBN 3-900051-07-0 Edition.

http://www.R-project.org

Reza, Fazlollah M., 1994. An introduction to information theory. Dover, New York, 496pp. 
Schabenberger, O., Gotway, C., 2004. Statistical Methods for Spatial Data Analysis, 1st Edition. Chapman \& Hall/CRC, 488pp.

Schlather, M. Riberio, P., Diggle, P., 2004. Detecting dependence between marks and locations of marked point process. Journal of Royal Statistical Society B (66), 79-93.

van Lieshout, M., Baddeley, A., 1999. Indices of dependence between types in multivariate point patterns. Scandinavian Journal of Statistics 26, 511-532.

Wagner, H., Fortin, M.-J., 2005. Spatial analysis of landscapes: Concepts and statistics. Ecology 86 (8), 1975-1987. 